\title{
Anomalies cellulaires de l'épithélium au cours des maladies inflammatoires cryptogénétiques de l'intestin
}

Par ses multiples fonctions l'épithélium intestinal joue un rôle central dans le maintien de l'homéostasie : il assure une barrière entre l'organisme et le monde extérieur, règle les mouvements d'eau et d'électrolytes, et contribue aux processus immunologiques de la muqueuse. Nombre de ces fonctions sont altérées chez les malades souffrant de maladies inflammatoires cryptogénétiques de l'intestin : augmentation de la perméabilité aux antigènes de la lumière intestinale et aux molécules pro-inflammatoires, survenue de diarrhée, altération apparente de la fonction immunologique. Les mécanismes pathogéniques d'origine muqueuse et luminale sont identifiés en partie, mais il reste difficile de différencier les anomalies épithéliales secondaires à l'inflammation, de celles qui surviennent au début du processus pathologique. La mise en évidence d'anomalies de l'épithélium spécifiques de la rectocolite hémorragique et indépendantes de l'inflammation de la muqueuse est en faveur d'un rôle central de l'épithélium colique dans le déclenchement de la maladie. En revanche, peu d'éléments suggèrent une anomalie primitive de l'épithélium dans la maladie de Crohn. L'étude des phénomènes physiopathologiques au niveau de l'épithélium intestinal devraient pouvoir conduire non seulement à une meilleure compréhension des mécanismes de la maladie, mais aussi à développer de nouvelles voies thérapeutiques.

'épithélium intestinal constitue une interface majeure entre le corps et le monde extérieur. Une de ses premières fonctions est de procurer une barrière physique entre le contenu de la lumière intestinale, imprévisible et potentiellement pathogène, et le milieu intérieur représenté par la lamina propria. Il n'est donc pas surprenant que l'épithélium joue un rôle dans la pathogénie des maladies inflammatoires intestinales. Le projet de cette revue est d'examiner les anomalies de l'épithélium répertoriées dans ces maladies, d'exposer dans leurs grandes lignes les mécanismes conduisant à ces désordres, et de 
tâcher de définir quel rôle joue l'épithélium dans la pathogénie de l'inflammation de la muqueuse ellemême dans la rectocolite hémorragique et la maladie de Crohn.

\section{Anomalies de l'épithélium}

\section{Anomalies morphologiques}

Les anomalies morphologiques de l'épithélium, à type d'ulcérations, de diminution de la sécrétion de mucus, de déformation des cryptes, sont manifestes en microscopie optique et électronique, s'associent à une inflammation de la muqueuse sous-jacente, ce qui n'a rien d'inattendu dans ce micro-environnement hostile. Dans la maladie de Crohn, la morphologie de l'épithélium est normale lorsque l'inflammation muqueuse a disparu histologiquement. Dans la rectocolite hémorragique, en revanche, les anomalies ultrastructurales telles que le raccourcissement des villosités, la vacuolisation des organites cytoplasmiques, la dilatation des espaces intercellulaires, sont présentes non seulement dans les phases de latence de la maladie, mais aussi dans la muqueuse apparemment saine [1]. Les modifications morphologiques s'accompagnent d'anomalies phénotypiques plus spécifiques ; de nombreuses anomalies de l'épithélium (expression d'antigènes de la membrane des cellules coliques, ou d'antigènes associés à des tumeurs) (pour revue voir [2]) sont communes à la rectocolite hémorragique aiguë et à la maladie de Crohn, mais quelques-unes sont spécifiques de la première, indépendantes de l'inflammation intestinale ou d'une atteinte antérieure.

\section{Profil métabolique}

Plusieurs observations clés ont fait porter l'attention sur le profil métabolique des cellules épithéliales du côlon [1]. Le butyrate est la principale source d'énergie de l'épithélium, avec une préférence remarquable par rapport aux autres substrats [3] ; c'est un acide gras à chaîne courte formé essentiellement au cours de la fermentation bactérienne des hydrates de carbone dans la lumière colique [2]. Lorsqu'un segment colique est mis hors du flux fécal, l'instillation régulière d'acides gras à chaîne courte dans la lumière permet d'évi- ter l'atrophie et l'inflammation de sa muqueuse [4]. Le butyrate serait donc un substrat essentiel pour le maintien de l'intégrité de l'épithélium colique et de sa fonction de barrière [3]. Dans la rectocolite hémorragique évolutive ou en rémission (et apparemment pas dans la maladie de Crohn) la capacité d'oxydation du butyrate par l'épithélium colique distal est diminuée [5]. Il faut noter que le traitement par des inhibiteurs de l'oxydation du butyrate provoque chez l'animal une colite qui ressemble à la rectocolite hémorragique [6]. Enfin, au cours de la rectocolite hémorragique, le jeûne métabolique, lié à une atteinte de cette voie métabolique, s'accompagne d'une inflammation muqueuse semblable à celle qu'on observe dans la colite par diversion* ; rappelons que, dans cette dernière, le jeûne métabolique, bien que de mécanisme différent (perte du substrat), semble être l'événement physiopathologique clé. La diminution de la capacité d'oxydation du butyrate a donc été proposée comme hypothèse pathogénique pour la rectocolite hémorragique $[2,5]$

Renouvellement des cellules de l'épithélium

Le renouvellement accéléré des cellules de l'épithélium, associé à l'inflammation muqueuse sous-jacentc, est un phénomène bien connu et non spécifique. Dans la rectocolite hémorragique, on observe aussi une augmentation de la prolifération épithéliale (sans allongement des cryptes) au sein d'une muqueuse rectale quiescente [7]. Des facteurs indépendants des processus inflammatoires muqueux contribuent donc sûrement à l'accélération du renouvellement cellulaire. On ne sait pas encore si ce phénomène est présent aussi au niveau de l'épithélium colique plus proximal, ou dans des segments apparemment sains.

\section{Études de cellules isolées de l'épi- thélium colique}

L'étude fonctionnelle de cellules isolées peut contribuer de façon importante à notre compréhension des caractéristiques biologiques de l'épi-

\footnotetext{
* Inflammation colique observée lorsque le côlon est exclu du courant fécal.
}

thélium dans les maladies inflammatoires cryptogénétiques de l'intestin (MICI). La capacité de la membrane plasmique des cellules des cryptes à retenir les protéines intracellulaires, mesurée par le taux de relargage du ${ }^{51} \mathrm{Cr}$ à partir de cellules préalablement marquées, est diminuée lorsque les cellules proviennent d'une muqueuse inflammatoire de maladie de Crohn ou de rectocolite hémorragique [8]. Cette anomalie est corrélée aux lésions de la membrane plasmique associées à l'inflammation de la muqueuse. Mais il faut noter que, dans la rectocolite hémorragique, plus de la moitié des cellules isolées d'une muqueuse quiescente à l'histologie, et un cinquième des cellules isolées d'une muqueuse apparemment saine, ont une fuite anormale de ${ }^{51} \mathrm{Cr}$; cela constitue une preuve supplémentaire des anomalies de l'épithélium, indépendante de l'inflammation (Tableau I). Les cellules des cryptes coliques isolécs ont une activité urokinase (probablement par association de l'urokinase à un récepteur), mais on n'a pas trouvé d'activité anormale chez les patients ayant une MICI [9]. L'urokinase liée à son récepteur pourrait jouer un rôle important dans la biologie de l'épithélium, telles la migration et l'ulcération cellulaires, et son taux est élevé dans la muqueuse des MICI [10]. Dans les conditions physiologiques, l'épithélium colique sécrète de l'urokinase (observations non publiées) et une anomalie de cette sécrétion et/ou de son contrôle pourrait avoir des conséquences pathogéniques voire thérapeutiques importantes.

\section{La barrière épithéliale intestinale}

L'inflammation de la muqueuse, quelle qu'en soit l'origine, est toujours associée à une augmentation de la perméabilité intestinale. Celle-ci a été mise en évidence dans les MICI à l'aide de sondes administrées par voie orale, telles que des sucres variés, le ${ }^{51} \mathrm{Cr}$-EDTA, ou le polyéthylèneglycol (PEG). Une autre preuve indirecte de l'anomalie de la perméabilité réside dans l'importance de la réactivité immunologique vis-àvis d'antigènes luminaux dans le sang périphérique, les ganglions lymphatiques mésentériques et la lamina propria (revue dans [2]). L'augmentation 


\section{Tableau}

ANOMALIES DE L'ÉPITHÉLIUM APPAREMMENT SPÉCIFIQUES DE LA RECTOCOLITE HÉMORRAGIQUE,

EN DEHORS D'UNE INFLAMMATION DE LA MUQUEUSE

\section{RÉFÉRENCES}

1. Delpre G, Avidor I, Steinherz R, et al. Ultrastructural abnormalitics in endoscopically and histologically normal and involved colon in ulcerative colitis. Am J Gastroenterol $1989 ; 84: 1038-46$.

2. Gibson PR, Pavli P. Pathogenic factors in inflammatory bowel discase. I. Ulcerative colitis. Dig Dis $1992 ; 10: 17-28$.

3. Roediger WEW. Role of anacrobic bacteria in the metabolic welfare of the colonic mucosa in man. Gut $1981 ; 21$ : 793-8.

4. Harig JM, Socrgel KH, Komorowski RA, et al. Treatment of diversion colitis with short chain-fatty acid irrigation. $N$ Engl J Med 1989 ; 320 : 23-8.

5. Roediger WEW. The colonic epithelium in ulcerative colitis : an encrgy-deficiency discasc. Lancet $1980 ; 2: 712-5$.

6. Roediger WEW, Nance S. Metabolic induction of experimental ulcerative colitis by inhibition of fatty acid oxidation. $\mathrm{Br} J$ Exp Pathol 1986 ; 67 : 773-82.

7. Allan A, Bristol JB, Williamson RCN Crypt cell production rate in ulcerative proctocolitis: differential increments in remission and relapse. Gut 1985; 26 : 999-1003.

8. Gibson PR, Van de Pol E, Barratt PJ, et al. Ulcerative colitis : a discase characterized by the abnormal colonic epithelial cell ? Gut $1988 ; 29$ : 516-21.

9. Gibson PR, Van de Pol E, Doc WF Cell-associated urokinase activity and colonic cpithelial cells in health and discasc. Gut 1991; $32: 191-5$

10. De Bruin PAF, Crama-Bohbouth F, Verspaget HW, et al. Plasminogen activators in the intestine of patients with inflammatory bowel discasc. Throm Haemost 1988 ; $60: 262-6$

11. Tysk C, Lindberg E, Jarnerot G, et al. Ulcerative colitis and Crohn's discase in an unselected population of monozygotic and dizygotic twins : a study of heritability and the influence of smoking. Gut $1988 ; 29$ : 990-6.

12. Hollander D, Vadheim CM, Brettholz $\mathrm{E}$, et al. Increased intestinal permeability in patients with Crohn's disease and their relatives. Ann Intern Med $1986 ; 105: 883-5$.

13. Katz KD, Hollander D, Vadheim CM, et al. Intestinal permeability in patients with Crohn's disease and their healthy relatives.

Gastroenterology 1989 ; 97 : 927-31.

de la perméabilité est-clle la cause et/ou l'effet de la maladie inflammatoire intestinale? On ne pourrait répondre de façon satisfaisante à cette question qu'en examinant la perméabilité intestinale au stade pré-clinique de la maladie. En effet, une fois la maladic installéc, il n'est malheureusement pas possible de prouver que l'intestin est normal dans sa totalité dans la maladie de Crohn, ni de différencier, dans la rectocolite hémorragique, la perméabilité anormale d'un épithélium apparemment sain de celle d'un épithélium apparemment cicatrisé. L'une des approches possibles, dans la maladie de Crohn, est l'étude de la parenté de premier degré, dans la mesure où la prédisposition génétique semble plus importante dans cette maladie (70 \% de concordance chez les jumeaux dizygotes [11]). Une anomalie spécifique de la perméabilité au PEG-400 a été décrite dans un groupe familial de ce type [12, 13], mais ces observations n'ont pu être reproduites par d'autres expérimentateurs. En ce qui concerne la rectocolite hémorragique, aucun élément ne permet d'établir de relation de cause à effet entre l'augmentation de la perméabilité intestinale et la maladie.

La barrière intestinale comporte un certain nombre de composants clés :

- Les jonctions serrées

$\mathrm{La}$ voie paracellulaire est une voie de passage importante lorsque la barrière principale entre les cellules épithéliales est assurée par des jonctions ser- rées. L'inflammation de la muqueuse augmente la perméabilité paracellulaire. On a identifié les facteurs responsables du réglage fonctionnel des jonctions serrées dans un modèle in vitro de l'épithélium intestinal, constitué de lignées de cellules T84. Il s'agit de facteurs solubles, l'interféron $\gamma$ et des facteurs de migration transépithéliale [14], mais il n'est pas sûr que ces résultats puissent être extrapolés à l'épithélium in vivo. Nos connaissances sur les jonctions serrées sont encore insuffisantes. Mais le développement rapide des connaissances sur les molécules d'adhérence cellulaire pourrait faire progresser notre compréhension des fonctions et dysfonctionnements des jonctions serrées.

\section{- La membrane plasmique}

Les macromolécules de la lumière intestinale peuvent traverser les cellules pour arriver à la lamina propria en pénétrant directement à travers la membrane cellulaire, par endocytose non spécifique ou relayée par un récepteur. L'importance de la voie de passage transcellulaire des macromolécules dans l'épithélium intestinal adulte est sans doute faible, comparée à celle qui prévaut dans l'intestin néonatal immature où la pinocytose est active. Bien qu'on ait mis en évidence, par des techniques ultrastructurales, une augmentation de l'activité de pinocytose dans la maladie de Crohn, on ne la retrouve que dans les phases aiguës de la maladie. Elle est donc vraisemblablement un 
phénomène secondaire. Dans la rectocolite hémorragique, aucune anomalie ultrastructurale ne permet de penser qu'il y ait passage transcellulaire anormal de macromolécules. Une sous-population de cellules épithéliales, spécialisées dans le transport rapide de macromolécules luminales vers les macrophages sous-jacents et les cellules dendritiques, recouvre les follicules lymphoïdes et les plaques de Peyer (épithélium associé aux follicules ou cellules M). Dans la mesure où les premières lésions dans la maladie de Crohn impliquent des réactions immunologiques à médiation cellulaire dans les follicules lymphoïdes, l'épithélium qui recouvre les follicules et leur est associé est le premier candidat pour fournir une porte d'entrée à l'antigène agresseur [15]. Cependant, aucun résultat ne permet de dire si le mécanisme qui conduit à ces réponses immunes anormales réside dans l'épithélium associé aux follicules lui-même, dans la nature de l'antigène ou dans la réponse immunologique qu'il suscite.

\section{- Le mucus}

Il réalise une barrière qui protège l'épithélium de multiples façons, en particulier par la formation d'un gel et par ses capacités de liaison. Ces propriétés appartiennent surtout aux mucines, qui sont des sialoglycoprotéines de grande taille, très glycosylées. La quantité de mucus produit dans les MICI n'est pas grossièrement anormale, mais une abondante littérature décrit les anomalies qualitatives des mucines (pour revue, voir [16]). De nombreuses anomalies de glycosylation portent sur la longueur des chaînes latérales et leur structure, analysées par histochimie, biochimie, liaison de lectines ou d'anticorps ; mais ces anomalies semblent être surtout des phénomènes secondaires. Il faut cependant noter que certaines anomalies sont relativement spécifiques à la rectocolite hémorragique, en particulier la réduction du contenu en sulfomucine, la baisse d'une des six fractions de glycoprotéine de la mucine colique, mise en évidence par chromatographie échangeuse d'ions (encore que l'interprétation de cette observation soit sujette à caution [17]), et l'expression de nouveaux épitopes antigéniques glycoprotéiques [18]. De plus, ces modifications sont $\mathrm{m} / \mathrm{s} n^{\circ} 8-9$ vol. 9, août-septembre 93 retrouvées indépendamment de l'état d'inflammation de la lamina propria sous-jacente et, au moins pour certaines, sont présentes dans la muqueuse apparemment saine (Tableau I). Ces perturbations sont-elles responsables de désordres fonctionnels, cela n'est pas clair, mais le taux réduit de sulfatation rend habituellement les mucines plus résistantes à l'attaque par les glycosidases. Les résidus acide sialique sont également des éléments protecteurs contre les glycosidases, mais la sensibilité aux sialidases est normale dans la rectocolite hémorragique. Fait intéressants (mais de signification incertaine), les mucines dans la maladie de Crohn ont une résistance accrue aux sialidases [16].

\section{- Immunité sécrétoire}

La libération à la surface de l'épithélium d'IgA sécrétoires (ou d'IgM), produites spécifiquement contre les antigènes luminaux échantillonnés dans les follicules lymphoïdes, réduit l'absorption des microbes ou des macromolécules présentant ces antigènes. Au cours de la maladie de Crohn, l'augmentation de la densité des cellules à $\mathrm{IgM}$ dans la lamina propria, associée à une muqueuse apparemment normale, indique (de façon indirecte) un déficit de la barrière IgA; la mesure plus directe de la production d'IgA sécrétoire par immunohistochimie [19], ou par culture d'organes [20], n'a pas montré de façon convaincante de déficit quantitatif d'IgA sécrétoire libérée dans l'intestin chez les malades atteints de maladie de Crohn. De même, est normal le taux de production des IgA sécrétoires par des cultures de muqueuse colique entière provenant de rectocolite hémorragique en phase de rémission [21].

\section{Mouvements d'eau et d'électrolytes} C'est une fonction importante de l'épithélium intestinal qui est souvent altérée dans les MICI, provoquant un excès d'eau fécale qui se traduit par la diarrhée. Ses mécanismes sont multiples et secondaires, pour une large part, à l'inflammation muqueuse elle-même. Quoi qu'il en soit, on trouve des anomalies spécifiques lors des poussées de rectocolite hémorragique - augmentation du chlorure et $\mathrm{pH}$ acide des fèces - qui reflètent la diminution d'excrétion du bicarbonate par l'épithélium colique [22]. Le mécanisme responsable est probablement la réduction de la production de $\mathrm{CO}_{2}$ provenant du métabolisme du butyrate ; on ne peut toutefois exclure une diminution de l'activité de l'anhydrase carbonique.

\section{Fonctions immunitaires}

L'épithélium, est à proximité immédiate des macrophages sousépithéliaux et des cellules dendritiques et est intimement lié aux lymphocytes intra-épithéliaux, dont le rôle reste mal élucidé. A l'état normal, les cellules de l'intestin grêle, et peut-être aussi celles du côlon, expriment les molécules du complexe majeur d'histocompatibilité $(\mathrm{CMH})$ de classe II ; elles sont capables de présenter l'antigène aux lymphocytes $T$ activés, in vitro, et de produire des facteurs de stimulation pour l'activation des lymphocytes $T[23,24]$. De plus, la réponse à cette présentation d'antigène conduit au développement de cellules suppressives non spécifiques de l'antigène, comme l'ont montré les études phénotypiques et fonctionnelles. Dans les MICI, l'expression du CMH de classe II par les cellules épithéliales augmente avec l'inflammation muqueuse sous-jacente [25], conséquence vraisemblable de l'exposition de ces cellules aux cytokines, en particulier à l'interféron $\gamma$. La fonction de présentation des antigènes de l'épithélium pourrait donc être stimulée, aidant ainsi à modérer la sévérité de la réaction immunitaire. Quoi qu'il en soit, les cellules intestinales épithéliales isolées de patients ayant une MICI induisent une expansion lymphocytaire de type auxiliaire (phénotype et fonction), que ces cellules proviennent d'une zone de la muqueuse saine ou pathologique [26]. Ces résultats surprenants posent plusieurs problèmes d'interprétation : par exemple, les cellules effectrices appartiennent au sang périphérique et non au secteur muqueux concerné ; il n'y a pas de spécificité d'organe (même chose pour le grêle et le côlon, même dans la rectocolite hémorragique) et les résultats déconcertants du traitement pharmacologique n'ont pas été exploités. De plus, l'expansion des lymphocytes auxiliaires n'est une caractéristique ni du secteur intra-épithélial, ni du secteur 


\section{RÉFÉRENCES}

14. Madara JL. Loosening tight junctions. Lessons from the intestine. J Clin Invest $1989 ; 83$ : 1089-94.

15. Pavli P, Gibson PR. Pathogenic factors in inflammatory bowel disease. 2. Crohn's disease. Dig Dis Sci 1993 (sous presse)

16. Rhodes JM. Mucus and inflammatory bowel disease. In : Allan RN, Keightley MRB, Alexander-Williams T, Hawkins CF Inflammatory Bowel Diseases, 2nd ed. Edimbourg : Churchill Livingstone, 1990, 171-9.

17. Raouf A, Parker N, Iddon D, et al. Ion exchange chromatography of purified colonic mucus glycoprotein in inflammatory bowel disease : absence of a selective subclass defect. Gut 1991; 32 : 1139-45

18. Podolsky DK, Fournier DA. Alterations in mucosal content of colonic glycocon jugates in inflammatory bowel disease defined by monoclonal antibodics. Gastroenterology $1988 ; 95$ : 379-81.

19. Kett K, Brandtzaeg P, Fansa (O. Jchain expression is more prominent in immunoglobulin A2 than in immunoglobulin A1 colonic immunocytes and is decreased in both subclasses associated with inflammatory bowel disease. Gastroenterology $1988 ; 94: 1419-25$.

20. McLelland DBL, Shearman DJC, Lai A Fat RFM, Van Furth R. In vitro synthesis of immunoglobulins, secretory component and lysozyme by human gastrointestinal tissues. Clin Exp Immunol 1976; 23 : 20-7.

21. Badr-El-Din S, Trejdosiewicz LK, Heatly RV, et al. Local immunity in ulcerative colitis : evidence for defective secretory IgA production. Gut $1988 ; 29: 1070-5$.

22. Roediger WEW, Lawson MJ, Kwok V, et al. Colonic bicarbonate output as a test of disease activity in ulcerative colitis. J Clin Pathol 1984; 37 : 704-7.

23. Bland PW, Warren L,G. Antigen presentation by epithelial cells of the rat small intestine. I. Kinetics, antigen specificity and blocking by anti-la sera. Immunology 1986 . $58: 1-7$.

24. Mayer I, Shlein R. Evidence for function of la molecules on gut epithelial cells in man. J Exp Med 1987; $166: 1471-83$.

25. Mclonald GB, Jewell DP. Class II antigen (HI,A-DR) expression by intestinal epithelial cells in inflammatory diseases of colon. J Clin Pathol 1987 ; 40 : 312-7. muqueux dans les MICI. Néammoins, ces observations semblent indiquer que l'épithélium potentialise les réponses inflammatoires de la muqueuse.

\section{Mécanismes à l'origine} des anomalies épithéliales (Tableau II)

\section{Facteurs muqueux}

- Facteurs solubles

Au cours de l'inflammation de la muqueuse, la libération de très nombreux facteurs solubles dans le liquide extracellulaire à proximité immédiate de l'épithélium crée un microenvironnement défavorable au maintien de son intégrité fonctionnelle. Si bien que les ulcérations, la déplétion du mucus et des mouvements anormaux d'eau et d'électrolytes sont fréquents. Il semble aussi que la libération de facteurs solubles dans la muqueuse normale soit liée à la légère inflammation chronique physiologique. Déméler lesquels de ces facteurs pourraient jouer un rôle important dans la pathogénie des anomalies de l'épithélium dans les
MICI n'est donc pas une tâche aisée. On a commencé à déterminer à quels types de facteurs solubles était exposé l'épithélium à l'état normal et au cours de l'inflammation, par la mesure des taux de ces facteurs et de leur ARNm dans les organes et en culture cellulaire. On a peu de données sur l'effet de ces facteurs sur la biologie de l'épithélium; quelques travaux ont mis en évidence une augmentation de la sécrétion de mucus et de liquide induite par l'histamine et certains eicosanoïdes, ainsi que l'expression accrue des antigènes de classe II du $\mathrm{CMH}$ et de la pièce sécrétoire induite par l'interféron $\gamma$ et le tumor necrosis factor $\alpha$. Les études sur lignées cellulaires apportent aussi des informations utiles, telles que l'augmentation de la perméabilité épithéliale (voir plus haut) relayée par l'interféron $\gamma$ et le tumor necrosis factor $\alpha$. Mais l'extrapolation de ces résultats à l'épithélium normal demande confirmation. Alternativement, l'épithélium des patients ayant une MICI pourrait répondre de façon anormale aux facteurs solubles, mais cet aspect a été peu étudié.

\begin{tabular}{|c|c|c|}
\hline $\begin{array}{r}\text { MÉCANISMES SUSCEP } \\
\text { DE L'ÉPITHÉLIUM AL } \\
\text { CRYPT }\end{array}$ & $\begin{array}{l}\text { Tableau ॥ } \\
\text { TIBLES D'ÊTRE À } \\
\text { COURS DES MA } \\
\text { OGÉNÉTIQUES DE }\end{array}$ & $\begin{array}{l}\text { INE DES ANOMALIES } \\
\text { INFLAMMATOIRES } \\
\text { ESTIN }\end{array}$ \\
\hline Muqueux & Intrinsèques & Luminaux \\
\hline Facteurs solubles & $\begin{array}{l}\text { Susceptibilité } \\
\text { génétique }\end{array}$ & $\begin{array}{l}\text { Infection des cellules } \\
\text { épithéliales }\end{array}$ \\
\hline $\begin{array}{l}\text { - médiateurs de } \\
\text { l'inflammation } \\
\text { - cytokines } \\
\text { - autres }\end{array}$ & & $\begin{array}{l}\text { produits du métabo- } \\
\text { lisme de la flore } \\
\text { microbienne }\end{array}$ \\
\hline $\begin{array}{l}\text { Atteinte immunitaire } \\
\text { spécifique } \\
\text { - auto-anticorps } \\
\text { - cellules } T \text { cytotoxiques }\end{array}$ & & Facteurs alimentaires \\
\hline $\begin{array}{l}\text { Cellules effectrices } \\
\text { naturelles } \\
\text { - cellules NK, LAK } \\
\text { - phagocytes }\end{array}$ & & \\
\hline $\begin{array}{l}\text { Facteurs } \\
\text { neurologiques } \\
\text { Ischémie }\end{array}$ & & \\
\hline
\end{tabular}


- Facteurs immunitaires

L'hypothèse physiopathologique du caractère auto-immun de l'attaque de l'épithélium dans la rectocolite hémorragique a eu un grand succès, mais le rôle des auto-anticorps, soit par action cellulaire directe, soit par cytotoxicité dépendante des anticorps, reste peu convainquant, malgré une littérature abondante sur le sujet depuis 33 ans [2]. L'implication des lymphocytes $T$ en tant qu'effecteurs pathogènes (comme dans beaucoup de maladies auto-immunes classiques) n'a été que très peu exploréc en raison de difficultés méthodologiques. Quoi qu'il en soit, des cellules effectrices appropriées sont présentes dans le compartiment muqueux, et la reconnaissance et la caractérisation d'auto-antigènes potentiels, telle la protéine $40 \mathrm{kDa}$ de la membrane basolatérale, pourraient faire progresser ce sujet [27].

- Les cellules effectrices naturelles I.es lymphocytes médiateurs de la cytotoxicité " naturelle" (cellules NK, LAK) ont été l'objet de beaucoup d'attention après l'observation, faite il y a 29 ans chez les malades souffrant de rectocolite hémorragique, de lymphocytes circulants actifs contre les cellules épithéliales coliques in vitro. Mais, malgré la présence de cellules NK et de précurseurs des cellules I.AK dans le compartiment muqueux, leur cytotoxicité envers un épithélium colique viable, normal ou provenant de patients avec MICI, n'est pas démontrable sur des systèmes in vitro quantitatifs et perfectionnés [2]. Des phagocytes pourraient avoir une action directe sur l'épithélium en plus de leur sécrétion de médiateurs de l'inflammation (radicaux libres, protéases). On a montré sur des lignées T84 que la migration trans-épithéliale des polynucléaires augmentait la perméabilité paracellulaire [14].

\section{- Autres facteurs}

Le système nerveux entérique peut altérer l'activité biologique de l'épithélium; la stimulation nerveuse sympathique peut, par exemple, moduler la vitesse de prolifération de l'épithélium intestinal [28]. La mise en évidence récente d'occlusion vasculaire dans les lésions inflammatoires de la maladie de Crohn, tant récentes que tardives, a donné un $\mathrm{m} / \mathrm{s} n^{\circ} 8-9$ vol. 9, août-septembre 93 regain d'intérêt au rôle de l'ischémie dans l'atteinte de l'épithélium [29].

\section{Facteurs luminaux \\ - Facteurs infectieux}

Actuellement, ni les études histologiques ni les études ultrastructurales n'ont apporté de preuve d'une atteinte infectieuse directe de l'épithélium intestinal par des bactéries ou des virus dans les MICI. De même, des micro-organismes connus pour leur caractère pathogène vis-à-vis des épithéliums n'ont que rarement été mis en évidence dans ces maladies. Le rôle d'E. coli dans la genèse des lésions épithéliales a été soupçonné, après qu'on a montré que des $E$. coli isolés des fèces des malades avaient des propriétés adhésives identiques à celles des souches entéropathiques [30], mais le caractère pathogène de ces bactéries et leur capacité de léser l'épithélium n'ont pas été démontrés. Des perturbations des caractéristiques métaboliques de la flore bactérienne colique pourraient cependant influencer profondément et de diverses manières la fonction épithéliale : (1) la fermentation bactérienne fournit un substrat énergétique indispensable (le butyrate) au bon fonctionnement de l'épithélium, mais la concentration de butyrate luminal est probablement normale, sauf sans les colites les plus sévères ; (2) les métabolites bactériens peuvent inhiber certaines voies métaboliques ; par exemple, le mercaptoacétate inhibe la $\beta$-oxydation du butyrate, et cette inhibition peut induire une colite chez l'animal d'expérience [6]. Mais on n'a pas encore montré d'augmentation de la production de mercapto-acétate dans les feces des malades ; (3) les enzymes bactériennes, glycosidases, sialidases, protéases, peuvent digérer les mucines et réduire l'effet protecteur de la couche de mucus. L'activité de la mucine sulfatase bactérienne est élevée dans les selles des malades souffrant de rectocolite hémorragique [31], ce qui explique la sulfatation basse de la mucine ; (4) la flore bactérienne peut produire en quantité excessive et potentiellement toxique des métabolites, comme les sulfures ; on a montré, dans les selles des malades souffrant de rectocolite hémorragique, des taux élevés de bactéries sulfo-réductrices et de production de sulfures [32]. Enfin, le pouvoir détoxifiant de molécules nuisibles de la flore bactérienne luminale est peutêtre réduit ; par exemple, la sulfatation des phénols par les bactéries est diminuée de façon importante dans les selles des malades souffrant de rectocolite hémorragique [33].

\section{- Facteurs alimentaires}

On connaît un certain nombre de facteurs alimentaires, ou apparentés, qui ont un effet vraisemblablement direct sur la perméabilité intestinale, mais sont diversement associés aux MICI. Par exemple, l'ingestion d'alcool est peu associée; les antiinflammatoires non stéroïdiens peuvent déclencher une petite inflammation intestinale, mais pas du type de celle de la maladie de Crohn; le tabac, qui réduit la perméabilité intestinale [34], a un rôle protecteur dans la rectocolite hémorragique et est un facteur de risque dans la maladie de Crohn. Le carragheen, composé alimentaire usuel qui induit une inflammation iléo-caecale chez l'animal de laboratoire, a des effets toxiques, in vitro, sur des monocouches de cellules de l'intestin grêle [35]. Avec des informations aussi pauvres, on ne peut ni infirmer ni affirmer le rôle direct des facteurs alimentaires sur la modulation de l'épithélium dans ces maladies.

\section{Anomalies intrinsèques de l'épithé- lium}

On envisage sérieusement la possibilité d'une anomalie génétique de l'épithélium depuis que l'on sait que des facteurs génétiques sont impliqués dans la pathogénie des MICI, en particulier dans la maladie de Crohn [11]. Cela dit, les données expérimentales sont rares. Le défaut spécifique de perméabilité du PEG-400, observé chez les parents apparemment sains de patients souffrant d'une maladic de Crohn [12], suggère typiquement qu'une anomalie épithéliale intrinsèque est à la base de la maladie. De nouvelles techniques d'isolement cellulaire permettant, par exemple, d'étudier les caractéristiques de l'épithélium apparemment sain dans la rectocolite hémorragique, l'utilisation des outils puissants de la biologic moléculaire, permettront peut-ĉtre d'identifier des anomalies spécifiques, déterminées génétiquement, suscepti- 


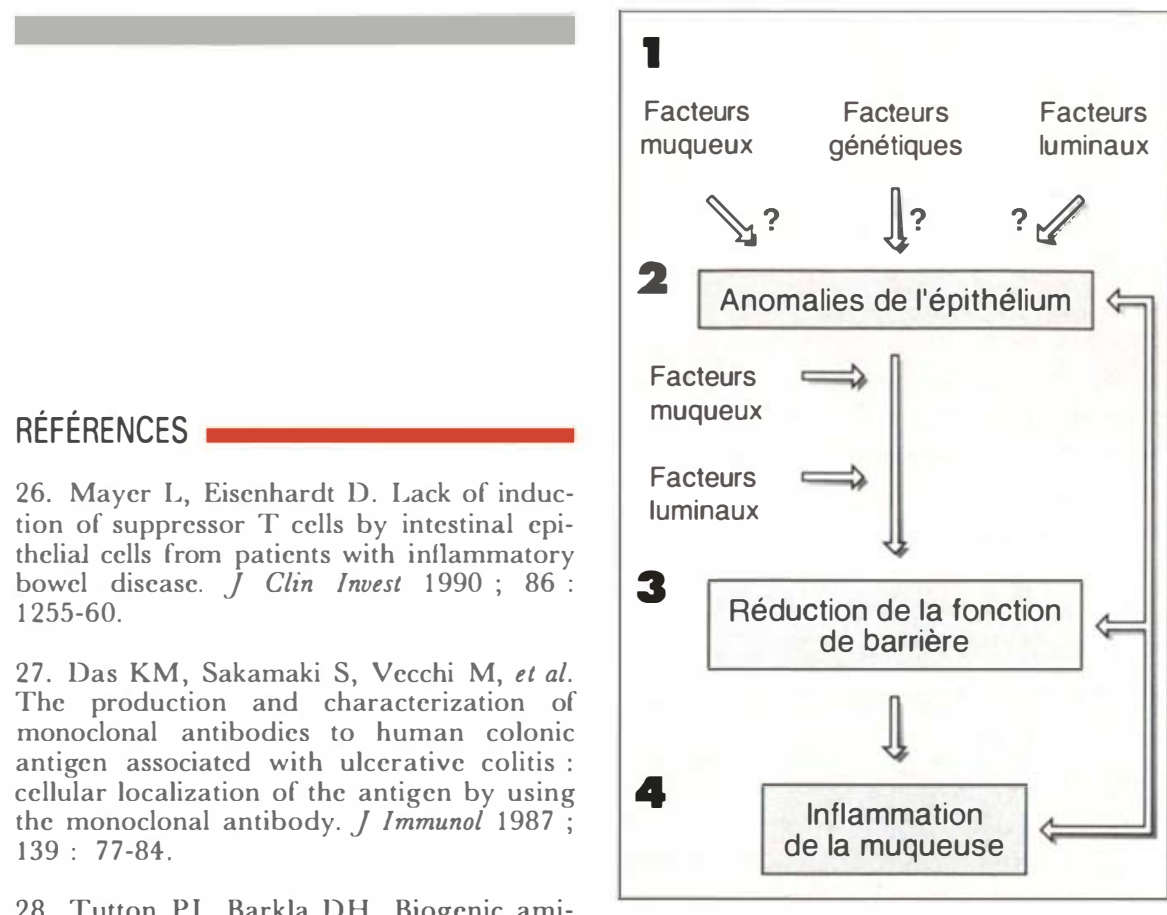

28. Tutton PJ, Barkla DH. Biogenic amines as regulators of proliferative activity of normal and neoplastic intestinal epithelial cells. Anticancer Res 1987; 7: 1-12.

29. Wakeficld AJ, Sawyer AM, Dhillon AP, et al. Pathogenesis of Crohn's discase : multifocal gastrointestinal infarction. Lancet $1989 ; 2$ : 1057-62.

30. Burke DA, Axon AT. Hydrophobic adhesion of $E$. coli in ulcerative colitis. Gut $1988 ; 29: 41-3$.

31. Tsai HH, Rhodes JM. Identification and characterization of a fecal mucin sulfatase with a possible pathogenic role in ulcerative colitis. Gastroenterology 1991; 100 : A621.

32. Florin THJ, Gibson GR, Neale $G$, et al. A role for sulfate reducing bacteria in ulcerative colitis ? Gastroenterology 1990 ; 98 : A 170 .

33. Ramakrishna BS, Roberts-Thompson IC, Pannall PR, Rocdiger WEW. Impaired sulphation of phenol by the colonic mucosa in quiescent and active ulcerative colitis. Gut 1991; 32 : 46-9.

34. Irvinc EJ, Lad R, Donnelly $\mathrm{M}$, St Louis P. Smoking habit and molecular volume alter pathways of permeation in Crohn's discasc. Gastroenterology 1991；100 : A218.

35. Ling KY, Bhalla D, Hollander D. Mechanisms of carragecnan in jury of IEC1 8 small intestinal epithelial cell monolayers. Gastroenterology 1988 ; 95 : 1487-95.

36. MacDonald TT, Horton MA, Choy MY, Richman PI. Increased expression of laminin/-collagen receptor (VLA-I) on cpithelium of inflamed human intestine. J Cin bles de jouer un rôle dans la pathogénie des MICI.

\section{Rôle de l'épithélium dans la pathogénie}

Rôle dans les effets pathogènes secondaires

L'épithélium joue clairement un important rôle secondaire dans la physiopathologie de l'inflammation muqueuse; l'inflammation entraîne une augmentation de la perméabilité intestinale qui expose la lamina propria à des molécules pro-inflammatoires qui, à leur tour, activent les réactions inflammatoires et immunologiques (figure 1). De plus, les interactions épithélium-lymphocytes, notamment en ce qui concerne la présentation antigénique, peuvent participer au déséquilibre de la balance immunorégulatrice puisque, au lieu d'une réponse normale (diminution des processus inflammatoires en induisant une suppression non spécifique de l'antigène), elles induisent les lymphocytes $T$ auxiliaires. Il est important de noter toutefois que les réponses épithéliales à l'inflammation ne sont pas toutes délétères, plusieurs réponses potentiellement favorables peuvent se produire, comme l'expression accrue de molécules d'adhérence (pour prévenir la perte cellulaire) [36].
Figure 1. Modèle pathogénique de la rectocolite hémorragique. (1) L'anomalie primitive, infraclinique, est un épithélium anormal de façon diffuse. (2) La cause de l'anomalie primitive est mal connue. (3) Des facteurs muqueux et/ou luminaux, qui ne sont pas forcément les mêmes que ceux qui sont responsables de l'anomalie primitive, peuvent provoquer un défaut de la fonction de barrière, probablement en induisant un déficit énergétique. (4) L'inflammation de la muqueuse est induite par l'afflux d'antigènes luminaux, de facteurs chimiotactiques, de facteurs immunitaires et de toxines consécutif à l'installation d'un défaut de barrière. Elle aggrave, à son tour, les anomalies de l'épithélium.

Rôle dans la rectocolite hémorragique

La présence d'une inflammation diffuse localisée à la muqueuse, associée à des anomalies morphologiques sévères de l'épithélium et des cryptes, de plusieurs anomalies épithéliales spécifiques de la rectocolite hémorragique indépendantes de l'inflammation muqueuse (et donc beaucoup moins susceptibles d'être des effets secondaires) (Tableau I) attire l'attention sur l'épithélium et amène à y chercher les premiers événements physiopathologiques de la rectocolite hémorragique. Dans notre esprit, la rectocolite hémorragique est une maladie de l'épithélium colique (figure 1). Les " lésions précoces " sont, par exemple, des changements morphologiques discrets, des modifications de la glycosylation de certaines glycoprotéines ou une réduction de la durée de vie de la cellule épithéliale colique. Ce n'est que lorsque la fonction de barrière de l'épithélium est compromise que l'inflammation muqueuse et les signes cliniques qui en découlent deviennent apparents. Les données exposées ci-dessus suggèrent que la déficience énergétique de l'épithélium, due à la défaillance de la $\beta$-oxydation des acides gras à chaîne courte, est un événement clé qui précipite la perte de sa fonction de barrière. La plus grande dépendance de l'épithé- 
lium colique tant distal que proximal vis-à-vis du butyrate comme substrat énergétique fournit une explication simple quant à la distribution topographique caractéristique de la rectocolite hémorragique. Les principes sur lesquels repose cette hypothèse sont montrés dans la figure 1. Les conséquences thérapeutiques sont nombreuses. La "thérapeutique de protection de l'épithélium ", telle qu'un apport adapté de butyrate à l'épithélium distal, la modulation de la flore intestinale ou l'utilisation de médicaments protecteurs de l'épithélium vis-à-vis des facteurs solubles peuvent fournir un certain nombre de nouvelles perspectives thérapeutiques qui restent à explorer.

\section{Rôle dans la maladie de Crohn}

La présence d'une atteinte diffuse de l'épithélium est incompatible avec la distribution par plaques de la maladie et l'atteinte transmurale et ganglionnaire. De plus, les lésions les plus précocement détectables dans la maladie de Crohn, les microgranulomes de la lamina propria, ne sont pas associées à des anomalies morphologiques de l'épithélium et ne sont pas infiltrés de polynucléaires qui sont généralement associés au flux de macromolécules luminales qui traversent une barrière épithéliale défectueuse. Il est beaucoup plus probable que, dans le début de l'affection, l'épithélium est impliqué par un défaut sélectif de la captation des macromolécules. Les éléments en faveur de ce mécanisme sont fragiles et reposent sur une constatation discutée : l'augmentation de perméabilité intestinale au PEG-400 (et non aux oligosaccharides) chez des parents sains de patients ayant une maladie de Crohn. L'association étroite de microgranulomes et d'ulcérations aphtoïdes aux follicules lymphoïdes sous-jacents suggère que, s'il $y$ avait un défaut épithélial, celui-ci intéresserait l'épithélium qui recouvre les follicules lymphoïdes. Il n'y a cependant pas de résultat à l'appui de cette hypothèse.

\section{Conclusions}

Au cours des maladies intestinales inflammatoires, le rôle que joue l'épithélium est primordial dans la surve-

$\mathrm{m} / \mathrm{s} \quad n^{\circ} 8-9$ vol. 9, août-septembre 93 nue de symptômes tels que la diarrhée et la genèse de l'inflammation muqueuse; il est probable dans des événements pathogéniques précoces de la rectocolite hémorragique, et possible dans la maladie de Crohn. Grâce à une meilleure compréhension des interactions importantes entre lumen, épithélium et système immunitaire muqueux, on s'éloigne d'une vision étroite et monodisciplinaire de ces maladies, pour aller vers une conception plus large associant l'immunologie, la biologie cellulaire et la microbiologie

\section{Summary}

Epithelial cell abnormalities in inflammatory bowel diseases

The intestinal epithelium plays critical roles in maintenance of the body's homeostasis due to its many functions such as providing a barrier to the outside world, handling of water and electrolytes, and contributing to mucosal immunological processes. Since many of these functions are perturbed in patients with inflammatory bowel diseases, leading to increased permeation of the barrier by luminal antigens and proinflammatory molecules, the generation of diarrhoea and an apparent disturbance of immunological accessory function, important pathogenic roles of the epithelium in inflammatory bowel diseases are likely. Although many putative mechanisms of mucosal and luminal origin which may underly these changes have been identified, the differentiation of epithelial abnormalities that are a consequence of inflammation from those that occur earlier in pathogenesis is problematical. Nevertheless, the identification of epithelial abnormalities occurring specifically in ulcerative colitis and independently of the presence of mucosal inflammation has suggested a pivotal role of the large bowel epithelium in early events during the pathogenesis of ulcerative colitis. Evidence that a primary abnormality of intestinal epithelium underlies Crohn's disease, however, remains scant. Increased knowledge of the pathobiology of the intestinal epithelium in inflammatory bowel diseases may lead not only to a greater understanding of disease pathogenesis but also to the development of novel therapeutic approaches. 